Conjugated ionic (co)polythiophene-based cathode interlayers for bulk heterojunction organic solar cells

Peer-reviewed author version

GOVAERTS, Sanne; KESTERS, Jurgen; Defour, Maxime; Van Mele, Bruno;

PENXTEN, Huguette; NEUPANE, Shova; RENNER, Frank; LUTSEN, Laurence;

VANDERZANDE, Dirk \& MAES, Wouter (2017) Conjugated ionic

(co)polythiophene-based cathode interlayers for bulk heterojunction organic solar cells. In: EUROPEAN POLYMER JOURNAL, 97, p. 49-56.

DOI: 10.1016/j.eurpolymj.2017.09.043

Handle: http://hdl.handle.net/1942/25560 


\section{Conjugated ionic (co)polythiophene-based cathode interlayers for bulk heterojunction organic solar cells}

Sanne Govaerts, ${ }^{\dagger}$ Jurgen Kesters, ${ }^{\dagger}$ Maxime Defour, ${ }^{\ddagger}$ Bruno Van Mele, ${ }^{\ddagger}$ Huguette Penxten, ${ }^{\dagger}$ Shova Neupane, ${ }^{\text {I }}$ Frank Uwe Renner, ${ }^{\text {ๆ, } ~}{ }^{\S}$ Laurence Lutsen, ${ }^{\S}$ Dirk Vanderzande, ${ }^{\dagger, \S}$ Wouter Maes*,†,§

${ }^{\dagger}$ UHasselt - Hasselt University, Institute for Materials Research (IMO), Design \& Synthesis of Organic Semiconductors (DSOS), Agoralaan, 3590 Diepenbeek (Belgium)

${ }^{\ddagger}$ Vrije Universiteit Brussel (VUB), Physical Chemistry and Polymer Science (FYSC), Pleinlaan 2, 1050 Brussels (Belgium)

"UHasselt - Hasselt University, Institute for Materials Research (IMO), Energy Materials and Interfaces (EMInt), Wetenschapspark 1, 3590 Diepenbeek (Belgium)

${ }^{\S}$ IMOMEC Division, IMEC, Wetenschapspark 1, 3590 Diepenbeek (Belgium)

Corresponding Author: E-mail: wouter.maes@uhasselt.be 


\section{Abstract}

The incorporation of conjugated polyelectrolytes as cathode interlayers in organic photovoltaics has been proven to be an effective way to boost the device efficiency. Nevertheless, more detailed investigations of the structure-property relationships of these interlayer materials, in particular related to the film deposition behavior, can provide further insights into their mode of action. With this aim, a series of ionic (co)polythiophenes is successfully synthesized via Kumada catalyst-transfer condensation polymerization and subsequent introduction of ionic moieties on the polymer side chains. Both the topology (i.e. homopolymers, random and block copolymers) and the amount of ionic groups are systematically varied. The polymers are fully characterized and then applied as cathode interlayers in polymer solar cells based on PCDTBT:PC 71 BM, affording an average efficiency increase of $~ 15 \%$. The structural screening on one hand indicates that the efficiency gain is a rather general phenomenon for this material class. On the other hand, the best photovoltaic responses are observed for the conjugated polyelectrolytes with a higher triethylene glycol side chain ratio and the block copolymer structure performs slightly better as compared to the random copolymer with the same (50/50) monomer ratio. Based on these findings, the field can move on to a more rational development of novel interfacial materials and thereby push the device efficiency even further.

Keywords: conjugated polyelectrolytes; polymer solar cells; interfacial materials; topology; structure-property relationships

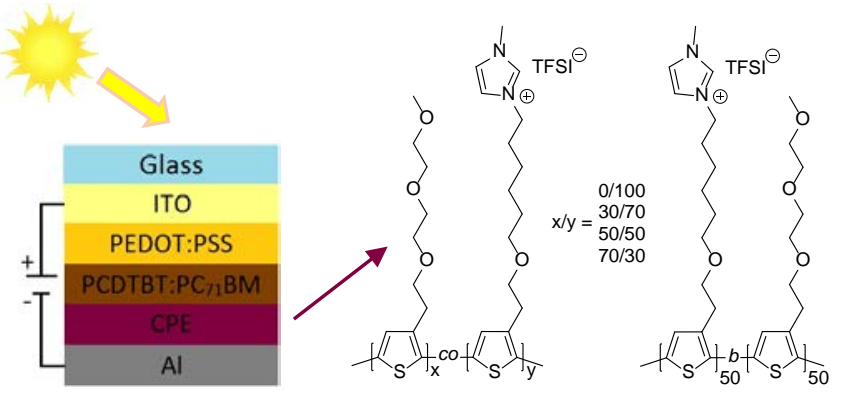




\section{Introduction}

Over the past decade, organic photovoltaics (OPVs) have shown a growing potential as an alternative renewable energy source, in particular because of some additional appealing features such as flexibility, semi-transparency, improved low-light performance, and low-cost (high-throughput) large area production. ${ }^{1-3}$ The introduction of the bulk heterojunction (BHJ) concept, in which the photoactive layer consists of electron donating and accepting materials intimately mixed at the nanoscale, the development of push-pull type low bandgap organic semiconductors to enhance the solar spectrum coverage, and the use of optimized device architectures have been key developments to elevate the power conversion efficiency (PCE) of OPV devices to values currently exceeding $12 \% .{ }^{4-8}$ Even though most research activities have been oriented on the development of novel active layer materials with appropriate optoelectronic properties, the introduction of interlayer materials has become a widely accepted approach to further enhance the device efficiency. ${ }^{9-17}$ One class of interlayer materials of particular interest are conjugated polyelectrolytes (CPEs). They can be processed from eco-friendly, orthogonal solvents, thereby preventing re-dissolution of the underlying layer during device fabrication. The incorporation of such CPEs has led to PCE improvements of more than $20 \%$ compared to reference devices, which can mainly be attributed to the

formation of interfacial dipoles, influencing the work function of the adjacent electrode. ${ }^{10,18-23}$ This improves the energy level alignment between the photoactive layer and the cathode (in standard device stacks), resulting in an enhanced charge collection and a reduced charge recombination.

Several CPE materials have already been studied as cathode interlayers. The conjugated polymer backbone has not been explored much further than polyfluorene and polythiophene derivatives and most variation has been introduced in the ionic moieties. ${ }^{12}$ Previous work in our group focused on imidazolium-functionalized polythiophenes, outperforming the 
ammonium-functionalized counterparts. ${ }^{11}$ More recently, impedance spectroscopy measurements revealed that the dielectric permittivity, induced by the ionic functionalities, is an important parameter to improve charge collection. ${ }^{23}$ The exact CPE structure also influences other aspects. An ionic random (50/50) copolythiophene with triethylene glycol and imidazolium-functionalized side chains (P3; Figure 1) for instance demonstrated superior performance in comparison to a fully imidazolium-functionalized homopolymer, resulting from an improved affinity with the photoactive layer beneath due to the presence of the nonionic side chains. ${ }^{23}$ For inverted OPV devices incorporating the CPE at the bottom side, Chen et al. demonstrated that more smooth and homogeneous photoactive layer deposition could be obtained by increasing the amount of polar groups in polyfluorene-based CPEs. ${ }^{22}$

In this work, a series of polythiophene CPEs with TFSI (bis(trifluoromethane)sulfonimide) counteranions was synthesized by Kumada catalyst-transfer condensation polymerization (KCTCP), subsequent substitution of the bromide-functionalized side chains, and final exchange of the Br counteranions. Due to the controlled chain-growth character of this type of polymerization, different polymer topologies (homopolymers, random and block copolymers) were obtained with a high control over the molar mass, dispersity and end groups in a relatively straightforward way via one-pot procedures. The ratio of the ionic groups present in the random copolymers was systematically varied and a block copolymer was prepared as well for comparison. The synthesized materials were then evaluated as cathode interlayer materials in standard BHJ OPV devices based on PCDTBT:PC ${ }_{71} \mathrm{BM}$ to further explore the effect of structural changes on the overall device performance. 


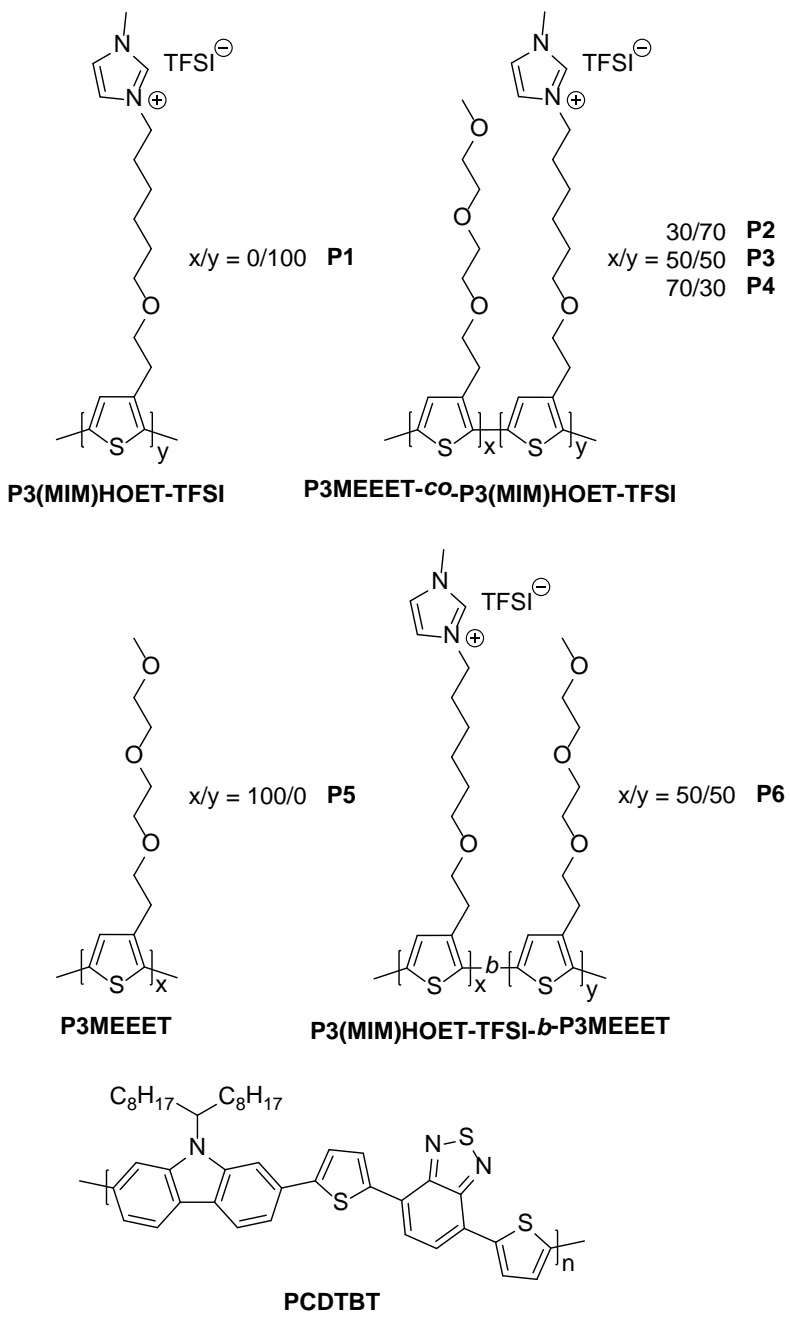

Figure 1. Overview of the synthesized polymers and the used donor material.

\section{Results and discussion}

\section{Polymer synthesis}

From previous work, the P3MEEET-co-P3(MIM)HOET-TFSI (50/50) random copolymer (P3; Figure 1) was identified as the CPE material affording best OPV device efficiencies for various photoactive layer blend combinations. ${ }^{23-25}$ This material was hence used as the starting point to create further structural variation. A series of random copolymers with monomer ratios varying from 0/100, 30/70, 50/50, 70/30 to 100/0 was targeted. For the synthesis of these random copolymers, the two different precursor monomers were first prepared, i.e. 2,5-dibromo-3-[2-(2-(2-methoxyethoxy)ethoxy)ethyl]thiophene (pM1) and 2,5- 
dibromo-3-[2-(6-bromohexyloxy)ethyl]thiophene (pM2) (Scheme 1). ${ }^{26}$ These precursor monomers were then activated via a Grignard metathesis (GRIM) reaction. This was done prior to the polymerization by combining $\mathbf{p M 1}$ and $\mathbf{p M} 2$ in the respective ratio in dry THF, whereby a monomer concentration of $0.1 \mathrm{M}$ was maintained, and adding 1.0 equivalents of $i$ PrMgCl.LiCl to this mixture at $0{ }^{\circ} \mathrm{C}$. After stirring for $1 \mathrm{~h}$, the active monomers $\mathbf{M} 1$ and $\mathbf{M} 2$ were obtained and transferred to a flask with the $\mathrm{Ni}(\mathrm{dppp}) \mathrm{Cl}_{2}$ catalyst in dry THF (monomer concentration $=0.075 \mathrm{M}$ ) to start the polymerization (Scheme 1). Due to the controlled character of the KCTCP, the amount of Ni-catalyst could be adjusted for each individual polymerization to always end up with a number-average molar mass $\left(M_{\mathrm{n}}\right)$ of $\sim 25000 \mathrm{~g} / \mathrm{mol}$.

Scheme 1. Synthesis of the ionic (co)polythiophenes via KCTCP and two post-polymerization reactions.
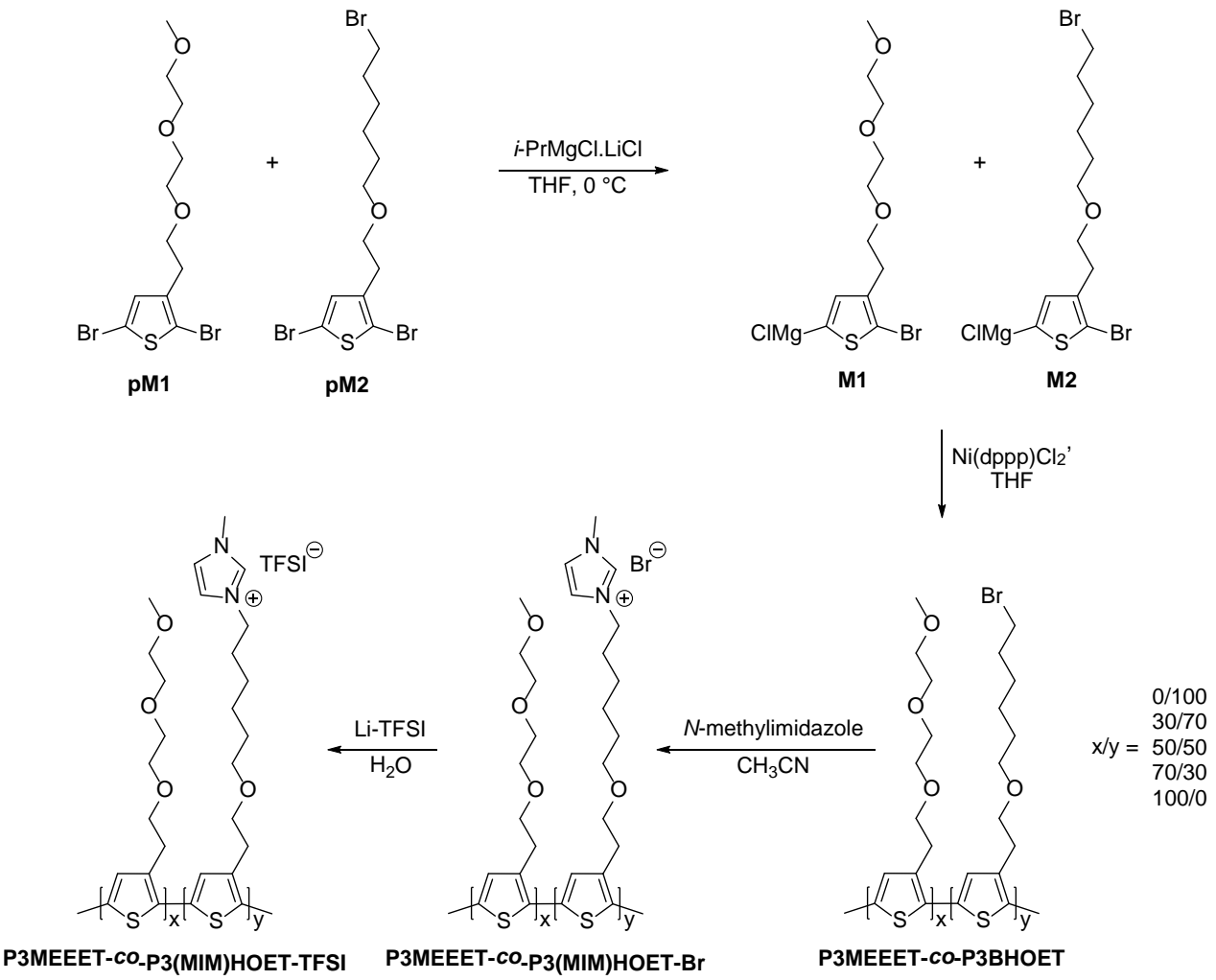

To optimize the polymerization conditions and to be able to use a uniform polymerization method for the synthesis of all ionic (co)polythiophenes, the polymerization conditions for the two homopolymers, P3BHOET (poly[3-(2-(6-bromohexyloxy)ethyl)thiophene-2,5-diyl]; x/y 
= 0/100) and P3MEEET (poly[3-(((methoxyethoxy)ethoxy)ethyl)thiophene-2,5-diyl]; x/y = 100/0) were investigated first. In a first polymerization test, M2 was polymerized at RT and aliquots of the polymerization mixture were taken after 30 minutes, 1 hour, 5 hours, and 21 hours, and quenched with a $\mathrm{MeOH} / \mathrm{HCl}$ mixture. These aliquots were then analyzed by gel permeation chromatography (GPC) and the results are shown in Figure 2a. From the GPC profiles it can be seen that a shoulder arises after 30 minutes, with a molar mass that is about two times the molar mass of the main peak, resulting in a rise in dispersity $(\nexists)$ from 1.14 to 1.34. The appearance of this shoulder can be explained by the occurrence of disproportionation, whereby two polymer chains are coupled to each other. ${ }^{27}$ To evaluate whether this is also the case for the polymerization of P3MEEET, M1 was also polymerized at RT and aliquots were taken after 30 minutes, 1 hour and 2 hours. The resulting GPC profiles (Figure 2b) once again show a shoulder arising after 30 minutes, leading to a rise in dispersity from 1.31 to 1.50 . 

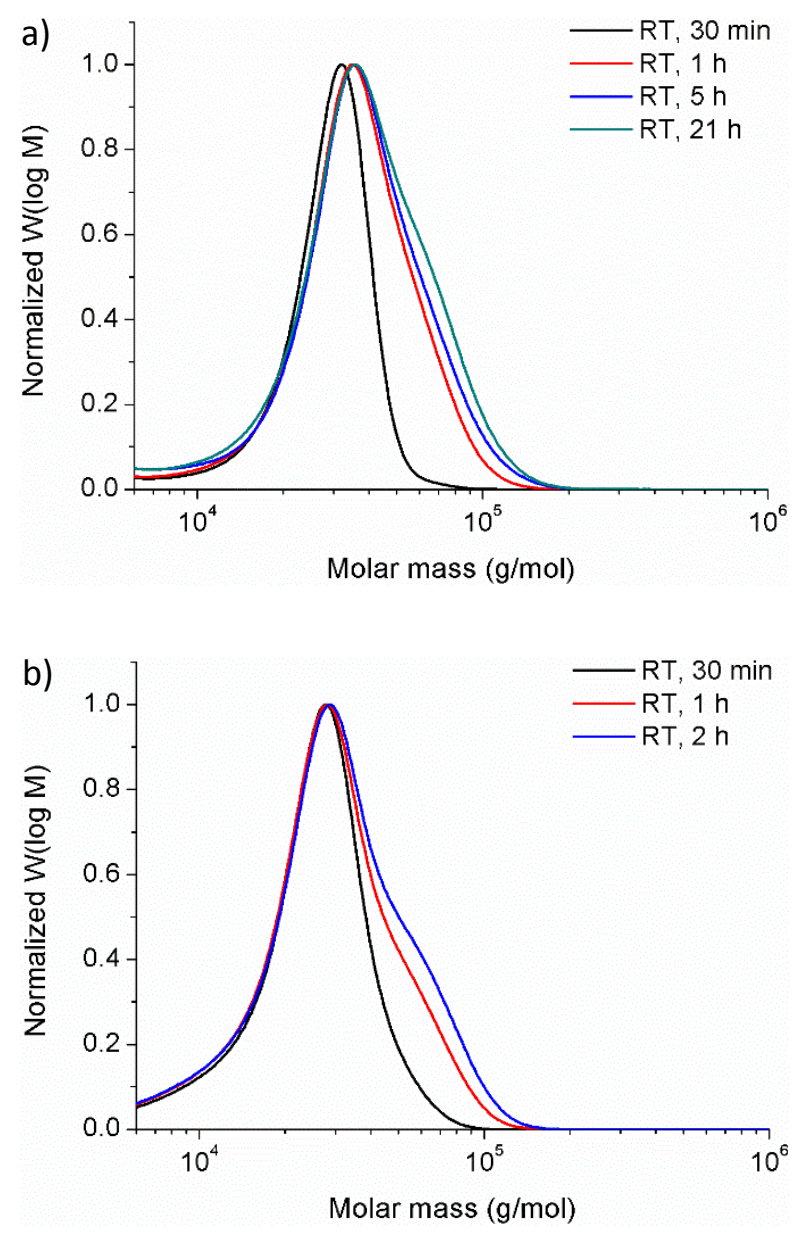

Figure 2. GPC profiles of the polymerization tests at RT of a) M2 and b) M1.

The above results indicated that a polymerization time of 30 minutes is optimal to obtain the desired molar mass and to avoid undesired termination reactions. Due to these findings, all polymerizations described in Scheme 1 were performed at RT and stopped after 30 minutes by quenching with a $\mathrm{MeOH} / \mathrm{HCl}$ mixture. Afterwards, the (co)polymers were purified by Soxhlet extractions with MeOH, acetone (for P3BHOET (0/100), P3MEEET-co-P3BHOET (30/70) and P3MEEET-co-P3BHOET (50/50)), hexanes, and chloroform, respectively. For the polymers with a higher P3MEEET content, it was not possible to use acetone for the Soxhlet extraction since these polymers readily dissolve in acetone. After final precipitation in MeOH, the P3MEEET-co-P3BHOET random copolymers were obtained as purple solids. The resulting molar masses and dispersities are listed in Table 1. The built-in ratios of the 
respective monomers were confirmed in the ${ }^{1} \mathrm{H}$ NMR spectra of the polymers (Figures S3-S6, Supporting Information).

Table 1. GPC data of the synthesized polymers.

$\begin{array}{ccc}\text { Polymer (x/y) } & M_{\mathrm{n}} \times 10^{4}(\mathrm{~g} / \mathrm{mol}) & \doteq \\ \text { P3BHOET (0/100) } & 1.7 & 1.18 \\ \text { P3MEEET-co-P3BHOET (30/70) } & 2.4 & 1.23 \\ \text { P3MEEET-co-P3BHOET (50/50) } & 2.8 & 1.16 \\ \text { P3BHOET-b-P3MEEET (50/50) } & 2.9 & 1.34 \\ \text { P3MEEET-co-P3BHOET (70/30) } & 2.6 & 1.17 \\ \text { P3MEEET (100/0) } & 1.9 & 1.30\end{array}$

After obtaining the random copolymers, two post-polymerization reactions were performed to obtain the desired ionic (co)polythiophenes (Scheme 1). In the first reaction, the bromide groups at the end of the alkyl chains were replaced by $N$-methylimidazolium moieties. This was done via reaction with a large excess of $N$-methylimidazole in acetonitrile under microwave irradiation for 4 hours at $100{ }^{\circ} \mathrm{C}$. The resulting ionic P3MEEET-coP3(MIM)HOET-Br (co)polymers were then precipitated in $\mathrm{Et}_{2} \mathrm{O}$ and purified by Soxhlet extractions in $\mathrm{Et}_{2} \mathrm{O}$ and $\mathrm{MeOH}$ (dissolving the polymers), respectively, to remove the excess of $N$-methylimidazole. This large excess of $N$-methylimidazole was used to ensure that all bromide entities were converted to $N$-methylimidazolium moieties, which was confirmed by ${ }^{1} \mathrm{H}$ NMR spectroscopy (Figures S7-S11, Supporting Information).

It was already shown before that ionic (co)polymers with bromide counteranions are strongly hygroscopic and as such not ideal for device incorporation. ${ }^{23,26,28}$ Therefore, the bromide counteranions were replaced by more hydrophobic TFSI counteranions in a final step. This was performed by dissolving the P3MEEET-co-P3(MIM)HOET-Br (co)polymers in water, followed by a dropwise addition of a Li-TFSI solution in water (Scheme 1). After a few hours, the resulting precipitates were filtered off over a cellulose membrane, washed with water and dried under vacuum. In this way, the desired ionic P3MEEET-co- 
P3(MIM)HOET-TFSI (co)polymers were finally obtained. Full counteranion exchange was confirmed by ${ }^{1} \mathrm{H}$ NMR spectroscopy.

Since the solar cell performance among others depends on the morphology of the interlayer material on top of the photoactive layer and block copolymers are known for their peculiar self-assembly properties, ${ }^{28-30}$ a block copolythiophene was synthesized as well from the same two monomer units in a 50/50 ratio (Scheme 2). This was done by first polymerizing the P3BHOET block by adding one equivalent of $i$-PrMgCl.LiCl to $\mathbf{p M 2}$ at $0{ }^{\circ} \mathrm{C}$, resulting in the active monomer $\mathbf{M} 2$ after $1 \mathrm{~h}$, and then adding this reaction mixture to $1.4 \mathrm{~mol} \%$ of $\mathrm{Ni}(\mathrm{dppp}) \mathrm{Cl}_{2}$ at RT. After a polymerization time of 15 minutes, a small fraction of the polymerization mixture was quenched with a $\mathrm{MeOH} / \mathrm{HCl}$ mixture, affording the P3BHOET reference homopolymer. To the rest of the polymerization mixture, the in situ prepared active monomer M1 was added to create the P3MEEET second block. The polymerization of this second block was allowed to further proceed for 30 minutes at RT, before being quenched with a $\mathrm{MeOH} / \mathrm{HCl}$ mixture. The resulting block copolymer was then purified by Soxhlet extractions with $\mathrm{MeOH}$, hexanes, and chloroform, respectively, finally yielding the P3ВHOET-b-P3MEEET block copolymer. 
Scheme 2. Synthesis of the ionic P3(MIM)HOET-TFSI-b-P3MEEET block copolymer.

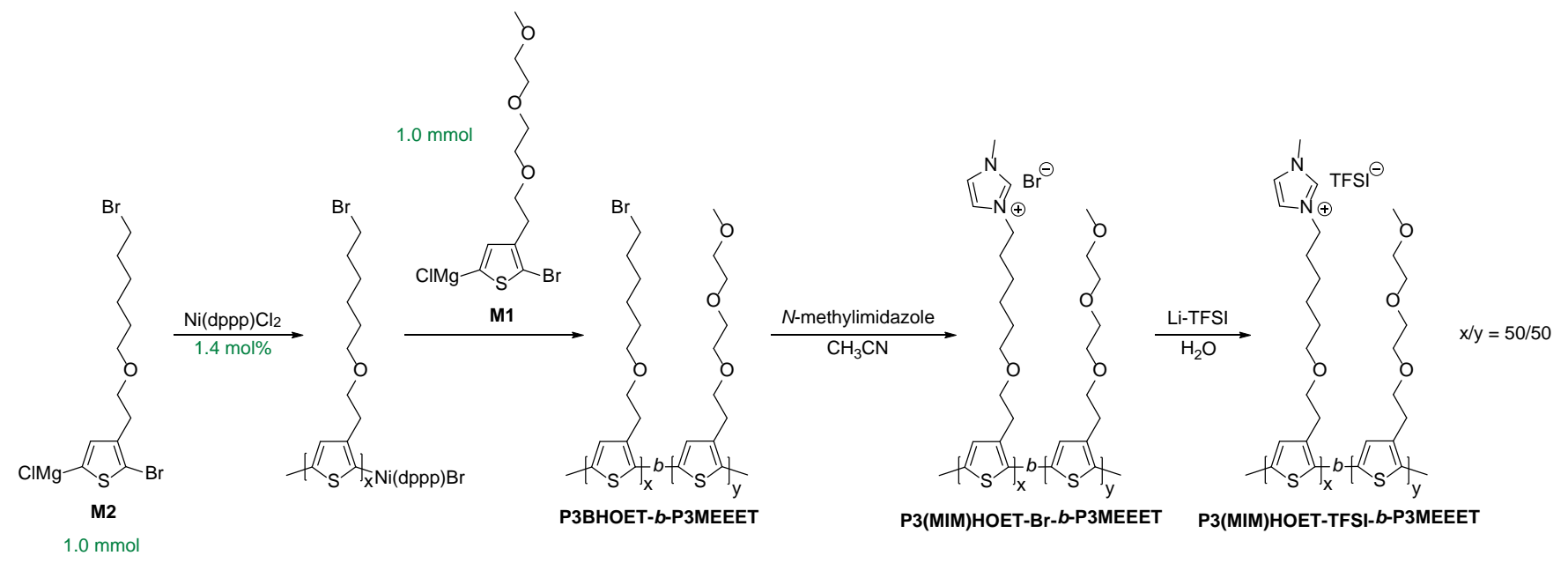

To verify the block copolymer formation, both the P3BHOET homopolymer sample and the P3BHOET-b-P3MEEET block copolymer were analyzed by GPC. From the resulting GPC profiles, it can be observed that there is a clear shift to higher $M_{\mathrm{n}}$ values for the block copolymer in comparison to the homopolymer (Figure 3). The P3BHOET homopolymer showed an $M_{\mathrm{n}}$ of $10000 \mathrm{~g} / \mathrm{mol}$ and a $Ð$ of 1.38, while the P3BHOET- $\boldsymbol{b}$-P3MEEET block copolymer has an $M_{\mathrm{n}}$ of $15000 \mathrm{~g} / \mathrm{mol}$ and a $Ð$ of 1.42 (before Soxhlet extraction). After Soxhlet extraction, the block copolymer showed an $M_{\mathrm{n}}$ of $29000 \mathrm{~g} / \mathrm{mol}$ and a $Ð$ of 1.34 (Table 1), nicely corresponding to the expectations and specifically targeted to be in range with the random copolymers described above. By using $1.4 \mathrm{~mol} \%$ of $\mathrm{Ni}(\mathrm{dppp}) \mathrm{Cl}_{2}$, an $M_{\mathrm{n}}$ of 26 000 g/mol ( 50 monomer units of $289 \mathrm{~g} / \mathrm{mol}$ for the P3BHOET block and 50 monomer units of $228 \mathrm{~g} / \mathrm{mol}$ for the P3MEEET block) was foreseen for the P3BHOET-b-P3MEEET block copolymer (taking into account a monomer loss of $25 \%$ due to the non-selective GRIM reaction). 


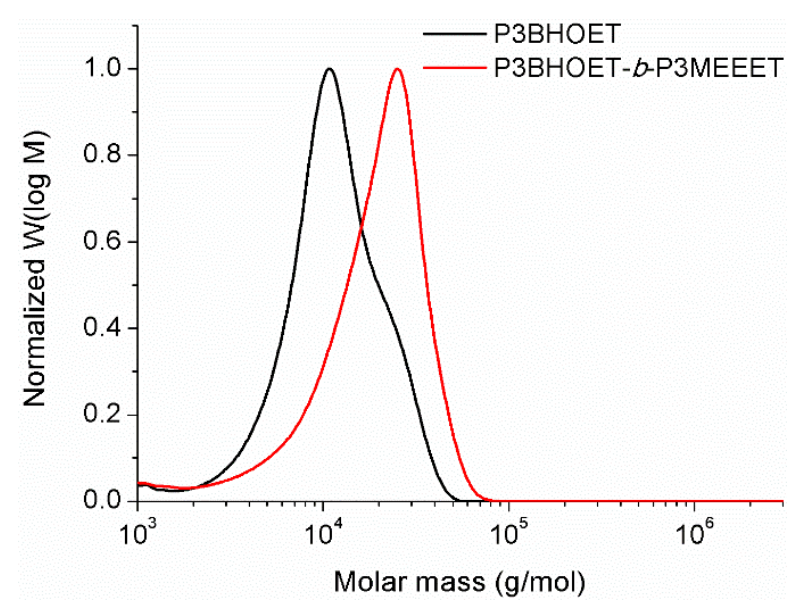

Figure 3. GPC profiles for the P3BHOET homopolymer (quench) and the P3BHOET-b-P3MEEET block copolymer (before Soxhlet extraction).

Finally, the obtained block copolymer was made ionic, following a similar procedure as described above, resulting into the ionic P3(MIM)HOET-TFSI-b-P3MEEET block copolymer (Scheme 2). An overview of the final synthesized ionic copolythiophenes is given in Figure 1. For the sake of clarity, the polymers are denoted as P1-P6, which will be used further on.

\section{Polymer characterization}

The optical properties of the synthesized polymers were investigated by UV-vis absorption spectroscopy. Because of the different amounts of incorporated ionic groups, the polymers showed varying solubility characteristics and different solvents had to be used. From the spectra in Figure 4, it is clear that all polymers show about the same absorption profile, with a wavelength at maximal absorbance $\left(\lambda_{\max }\right)$ located between 438 and $446 \mathrm{~nm}$. 


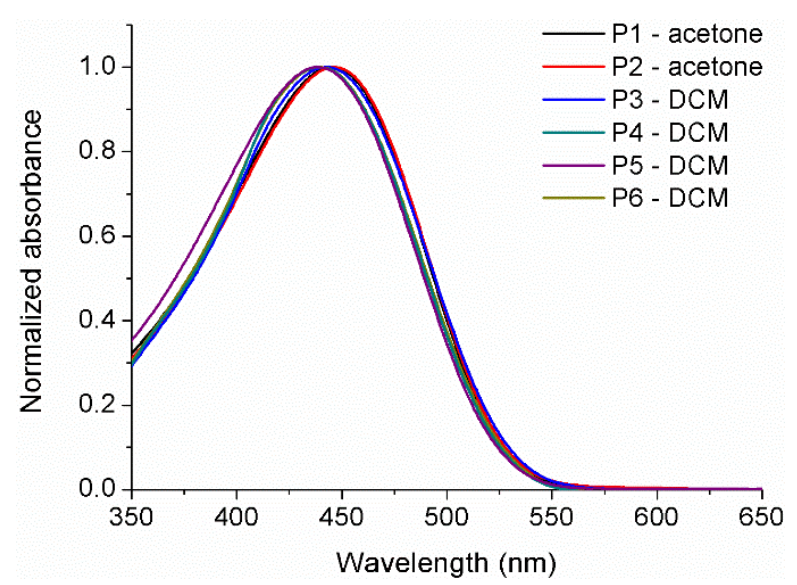

Figure 4. UV-vis absorption spectra in solution for the synthesized ionic polythiophenes P1-P6.

The electrochemical properties of the novel materials were analyzed by cyclic voltammetry (Table S1 and Figure S17, Supporting Information). P3HT-like behavior was observed for all materials. Detailed comparison of the obtained oxidation and reduction onsets and resulting HOMO-LUMO values is, however, complicated by the different solubility characteristics, impeding analysis of the full polymer series in one single solvent or in film.

The thermal stability of all polymers was studied by thermogravimetric analysis (TGA), showing strong similarities for all polymers and no significant mass loss up to $325-350{ }^{\circ} \mathrm{C}$ (Figure S18, Supporting Information). Further detailed thermal analysis was performed by rapid heat-cool calorimetry (RHC) measurements (Figure 5; Table S2-S3 and Figure S19, Supporting Information). From these data, it is clear that the $\mathbf{P 1}$ ionic homopolymer shows a relatively low glass transition temperature $\left(T_{\mathrm{g}}\right)$ and a low melting enthalpy $(\Delta H)$ after the first heating cycle. This melting behavior is, however, no longer observed when the polymer is reheated (second heating at $500 \mathrm{~K} / \mathrm{min}$ ) after a preceding cooling step at a relatively low cooling rate $(20 \mathrm{~K} / \mathrm{min})$, implying that this cooling rate is too high to allow the development of a crystalline phase. On the other hand, the fully TEGylated (TEG = triethylene glycol) P5 homopolymer shows a clear melting behavior after the first as well as the second heating cycle. The crystallinity of the random copolymers P2-P4 and the block copolymer P6 
depends on the relative content of the two constituent monomers. By decreasing the content of P3(MIM)HOET-TFSI, which clearly has a lower $\Delta H$ than P3MEEET, the melting enthalpy (and therefore the crystallinity) of the random copolymers increases. This is confirmed when the $\Delta H$ values of $\mathbf{P 2 - P 4}$ and $\mathbf{P 6}$ are estimated based upon the relative content of the two monomers and the $\Delta H$ values of the homopolymers $\mathbf{P 1}$ and $\mathbf{P 5}$ after the first heating cycle (Table S2, Supporting Information). The melting temperatures $\left(T_{\mathrm{m}}\right)$ and $\Delta H$ values after the second heating cycle are consistently lower than after the first heating cycle (Tables S2 and S3, Supporting Information) which could be explained by the continued development of stable crystals during casting or at room temperature. Another important observation is that the 50/50 random copolymer P3 and the 50/50 block copolymer P6 show very different heating profiles. The block copolymer shows a very broad melting behavior with two different peaks, while the random copolymer shows just one distinct melting peak located in between those of the two homopolymers $\mathbf{P 1}$ and P5 (Figure 5a). This difference further confirms the formation of a block copolymer. 

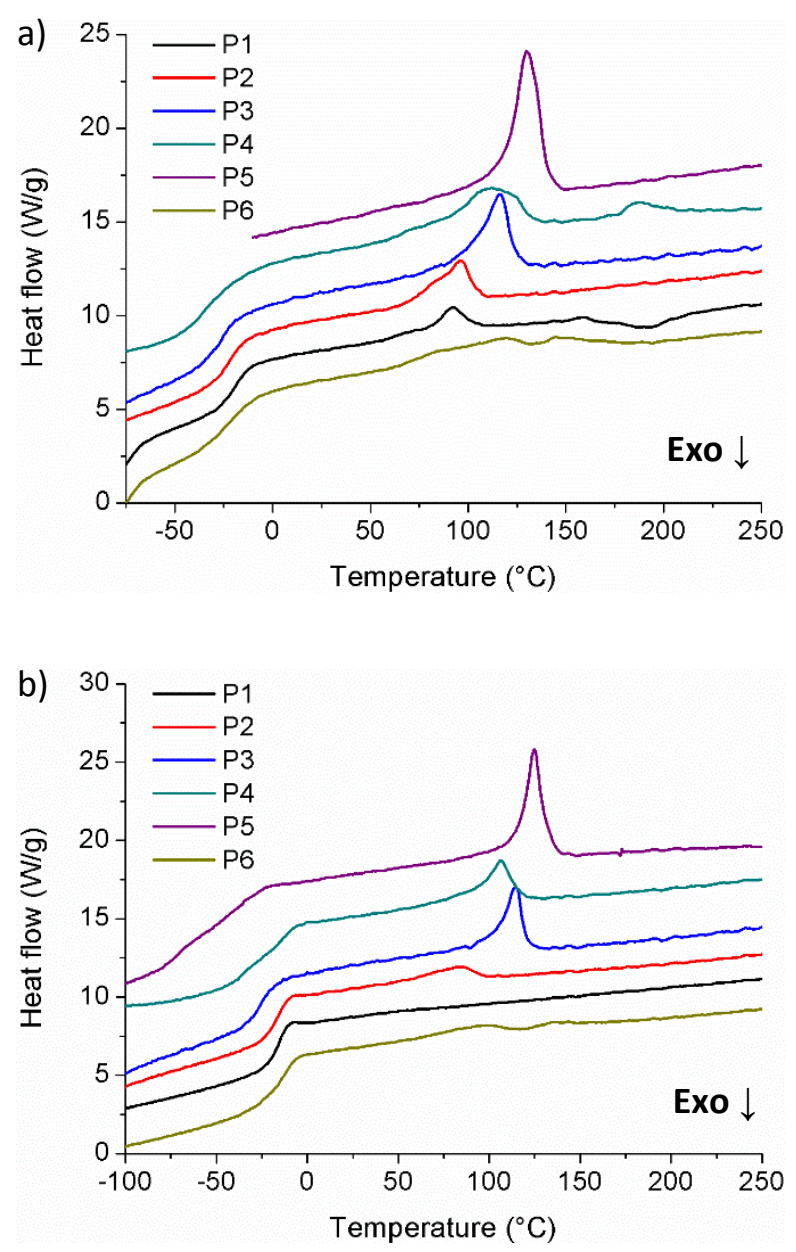

Figure 5. RHC heating profiles of the synthesized polymers P1-P6, obtained after a) a first heating cycle at $500 \mathrm{~K} / \mathrm{min}$, and b) a second heating cycle at $500 \mathrm{~K} / \mathrm{min}$ (after preceding cooling at $20 \mathrm{~K} / \mathrm{min}$ ). The curves are shifted vertically for clarity.

\section{Photovoltaic properties}

The incorporation of CPE interlayers in OPV devices has already been proven to be a successful strategy to boost the performance. In past work, we briefly demonstrated how the chemical structure of polythiophene-based CPE materials can influence the deposition behavior on top of different donor:acceptor blends. ${ }^{23}$ To gain further insights into the film formation properties, the newly synthesized CPE materials were incorporated in BHJ polymer solar cells with a standard device architecture consisting of glass/ITO/PEDOT:PSS/PCDTBT:PC 71 BM/interlayer/Al (the PCDTBT structure is depicted 
in Figure 1). The photoactive layer was prepared according to a literature recipe ${ }^{31}$ with a total concentration of $20 \mathrm{mg} / \mathrm{mL}$ in ortho-dichlorobenzene (oDCB) and a 1:4 polymer:PC ${ }_{71} \mathrm{BM}$ ratio. The CPEs were deposited on top of the active layer from methanol solutions with varying concentrations. The device results are summarized in Table 2 and Figure 6a (the corresponding dark curves are depicted in Figure S20, Supporting Information). Polymer P5 was excluded from the series because of its insoluble nature in the chosen solvent. As can be observed, the reference device employing calcium instead of a CPE exhibits an average PCE of $4.10 \%$, with a top efficiency of $4.34 \%$. Similar to previous studies on PCDTBT:PC ${ }_{71} B M$ devices containing CPE interlayers, ${ }^{11,23}$ all photovoltaic parameters (open-circuit voltage ( $\left.V_{\mathrm{OC}}\right)$, short-circuit current density $\left(J_{\mathrm{SC}}\right)$, fill factor $(\mathrm{FF})$, and PCE) were enhanced, resulting in an average PCE increase of 15-17\%. As such, the top performing device incorporating a CPE (P4) demonstrated, on average, a $V_{\mathrm{OC}}$ of $0.88 \mathrm{~V}$ (vs. $\left.0.84 \mathrm{~V}\right)$, a $J_{\mathrm{SC}}$ of $9.02 \mathrm{~mA} / \mathrm{cm}^{2}(v s$. $8.71 \mathrm{~mA} / \mathrm{cm}^{2}$ ) and a FF of $62 \%$ (vs. 56\%), resulting in an average PCE of $4.92 \%$ (vs. $4.10 \%$ ). The increase in $J_{\mathrm{SC}}$ for all interlayer containing devices was also confirmed by extracting the currents from external quantum efficiency (EQE) measurements on average-performing devices (Figure 6b), with $J_{\mathrm{EQE}}$ values of 8.00 , 8.81, 8.57, 8.44, 8.87, and $8.51 \mathrm{~mA} / \mathrm{cm}^{2}$ for the devices employing Ca, P1, P2, P3, P4, and P6, respectively (with corresponding $J_{\mathrm{SC}}$ values of 8.14, 8.79, 8.78, 8.77, 8.86, and $8.62 \mathrm{~mA} / \mathrm{cm}^{2}$, respectively).

Table 2. Photovoltaic parameters of BHJ polymer solar cells (PCDTBT:PC ${ }_{71} \mathrm{BM}$ ) with and without CPE cathode interlayers.

\begin{tabular}{cccccccc} 
Entry & $\begin{array}{c}\text { Cathode } \\
\text { Interlayer }\end{array}$ & $\begin{array}{c}\text { Concentration } \\
(\mathrm{mg} / \mathrm{mL})\end{array}$ & $\begin{array}{c}V_{\mathrm{OC}} \\
(\mathrm{V})\end{array}$ & $\begin{array}{c}J_{\mathrm{SC}} \\
\left(\mathrm{mA} / \mathrm{cm}^{2}\right)\end{array}$ & $\begin{array}{c}\mathrm{FF} \\
1\end{array}$ & $\begin{array}{c}\text { Average } \eta \\
(\%)^{a}\end{array}$ & $\begin{array}{c}\text { Best } \eta \\
(\%)\end{array}$ \\
-2 & $/$ & 0.84 & 8.71 & 0.56 & 4.10 & 4.34 \\
\hline 2 & $\mathbf{P 1}$ & 0.25 & 0.85 & 8.41 & 0.60 & 4.29 & 4.65 \\
3 & $\mathbf{P 1}$ & 0.5 & 0.87 & 8.90 & 0.58 & 4.49 & 4.93 \\
4 & $\mathbf{P 1}$ & 1 & 0.88 & 9.01 & 0.59 & 4.68 & 4.86 \\
5 & $\mathbf{P 1}$ & 1.5 & 0.86 & 9.09 & 0.61 & 4.77 & 5.11 \\
\hline 6 & $\mathbf{P 2}$ & 0.25 & 0.87 & 8.92 & 0.61 & 4.73 & 5.04 \\
7 & $\mathbf{P 2}$ & 0.5 & 0.88 & 8.86 & 0.61 & 4.76 & 4.76
\end{tabular}




\begin{tabular}{cccccccc}
8 & P2 & 1 & 0.87 & 8.94 & 0.60 & 4.67 & 4.91 \\
9 & P2 & 1.5 & 0.87 & 9.04 & 0.61 & 4.80 & 4.98 \\
\hline 10 & P3 & 0.25 & 0.84 & 8.35 & 0.62 & 4.35 & 4.38 \\
11 & P3 & 0.5 & 0.86 & 8.76 & 0.61 & 4.60 & 4.83 \\
12 & P3 & 1 & 0.87 & 8.86 & 0.61 & 4.70 & 4.81 \\
13 & P3 & 1.5 & 0.87 & 8.56 & 0.61 & 4.54 & 4.96 \\
\hline 14 & P4 & 0.25 & 0.86 & 8.32 & 0.63 & 4.51 & 4.80 \\
15 & P4 & 0.5 & 0.85 & 8.35 & 0.61 & 4.33 & 4.64 \\
16 & P4 & 1 & 0.88 & 9.02 & 0.62 & 4.92 & 5.11 \\
17 & P4 & 1.5 & 0.88 & 8.98 & 0.61 & 4.82 & 5.05 \\
18 & P6 & 0.25 & 0.88 & 9.23 & 0.60 & 4.87 & 5.06 \\
19 & P6 & 0.5 & 0.88 & 9.21 & 0.60 & 4.86 & 5.14 \\
20 & P6 & 1 & 0.88 & 9.39 & 0.59 & 4.84 & 4.95
\end{tabular}

${ }^{a}$ Average PCE over 8-16 devices.
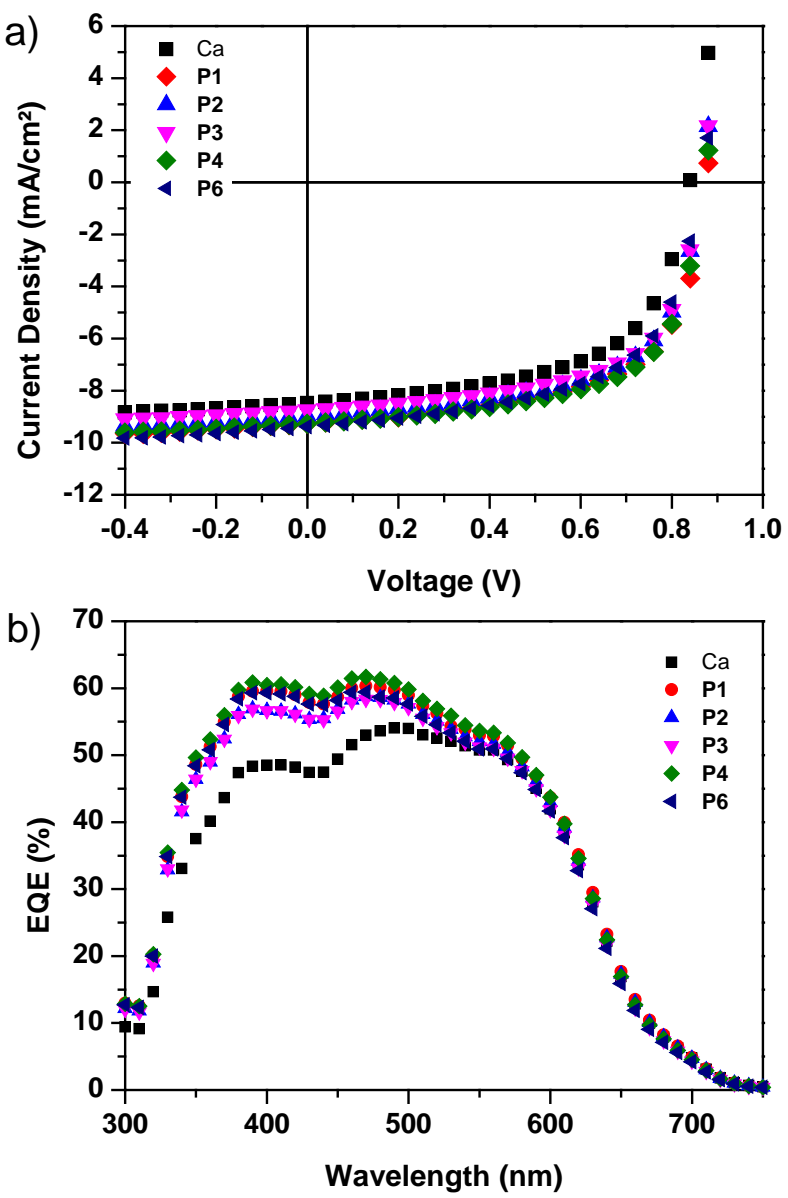

Figure 6. a) $J$ - $V$ curves under illumination for average performing PCDTBT:PC ${ }_{71} \mathrm{BM} B \mathrm{BH}$ photovoltaic devices with and without CPE cathode interlayers. b) EQE spectra of average performing devices bearing Ca and CPE interlayers P1-P6. 
To investigate the interlayer deposition behavior in more detail, atomic force microscopy (AFM) imaging was performed on samples containing the CPE materials deposited on top of the PCDTBT:PC 71 BM active layer (Figure 7; the entry numbers from Table 2 were added for convenience). Similar to previous observations, ${ }^{11,23}$ the polythiophene-based CPE interlayers never afford a completely covered layer, and the deposition patterns strongly vary depending on the CPE concentration (in methanol). Firstly, increasing the ratio of TEG-to-ionic side chains in the respective CPE materials results in an improved compatibility for deposition onto the photoactive layer blend, most profoundly observable for the samples with CPEs deposited from a concentration of $0.25 \mathrm{mg} / \mathrm{mL}$. Moreover, especially for $\mathbf{P} 3$ and $\mathbf{P} 4$, the lower device performances for the lower concentrations can be related to too narrowly distributed CPE domains (entries 10, 14 and 15), whereas slightly larger and interconnected domains appear to give rise to more elevated performances (entries 4, 5, 16 and 17). Furthermore, alteration of the chemical structure from the random copolymer $\mathbf{P} 3$ to the block copolymer P6, with an identical TEG-to-ionic side chain ratio, also influences the photovoltaic parameters. Solar cells employing the $\mathbf{P 6}$ interlayer demonstrated slightly superior performances across the entire concentration range in comparison to $\mathbf{P} 3$, mostly originating from an elevated $J_{\mathrm{SC}}$ (from an average of 8.65 to $9.28 \mathrm{~mA} / \mathrm{cm}^{2}$ ), finally resulting into PCEs exceeding 5\%. AFM imaging, however, did not reveal noteworthy differences between the deposition behavior of these two CPE interlayers. 


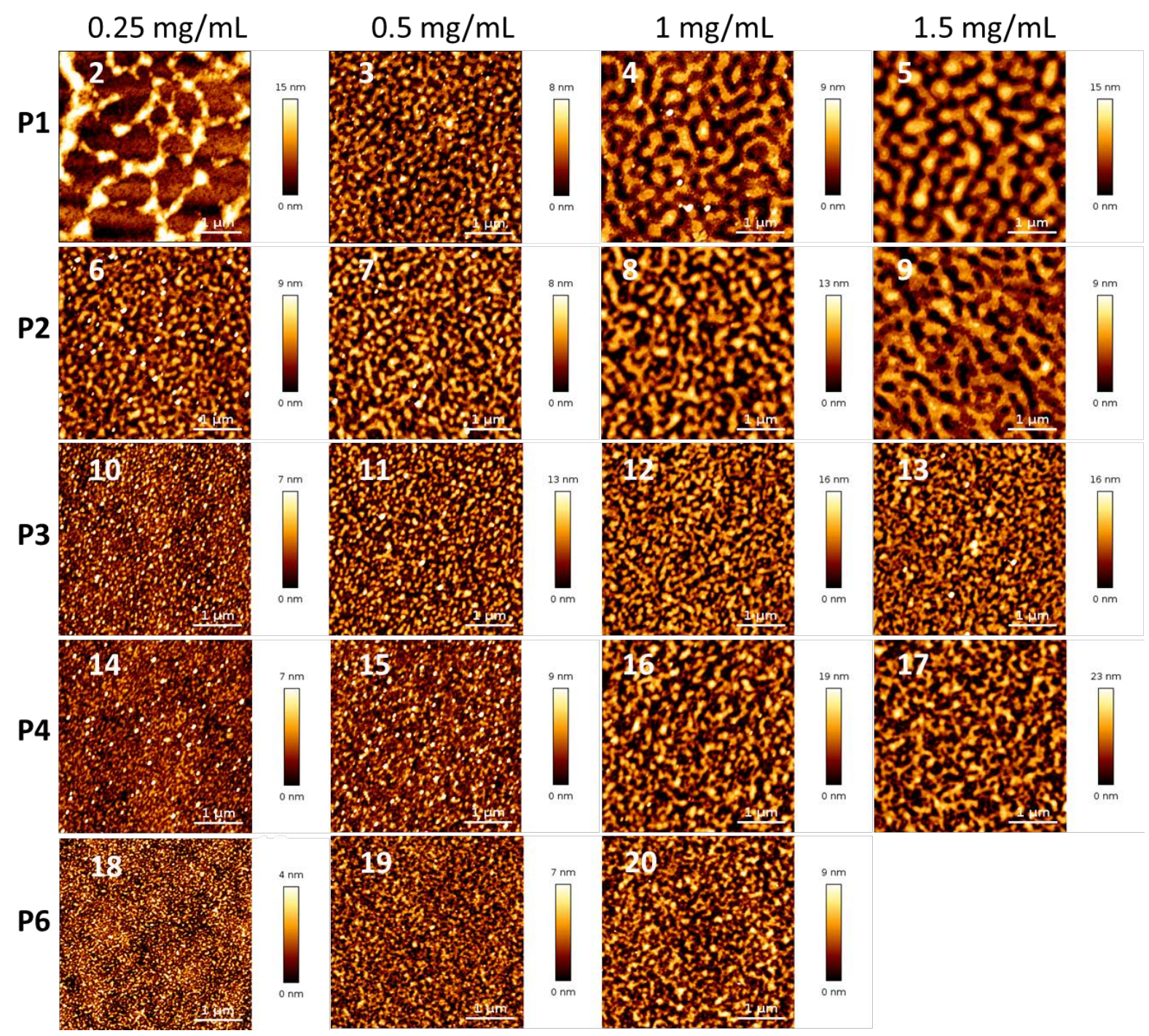

Figure 7. Atomic force microscopy images $(4 \times 4 \mu \mathrm{m})$ of CPE materials P1-P4 and P6 deposited from various concentrations in methanol on top of the PCDTBT:PC ${ }_{71} \mathrm{BM}$ active layer. The image numbers refer to the entries in Table 2.

In previous work, we demonstrated that the polythiophene-based CPE interlayers form a capacitive double layer due to the presence of the ionic moieties, thereby enhancing the dielectric constant and charge carrier dissociation of the overall solar cell. ${ }^{23}$ As a complementary technique, the charge dissociation probability $(P)$, determined by the correlation between the photocurrent $J_{\text {ph }}$ and the effective voltage $V_{\text {eff, can provide further }}$ insights into the exciton dissociation. ${ }^{32}$ Herein, $J_{\mathrm{ph}}$ can be defined as $J_{\mathrm{L}}-J_{\mathrm{D}}$, in which $J_{\mathrm{L}}$ and $J_{\mathrm{D}}$ are the current densities under light and dark conditions, respectively, and $V_{\text {eff }}$ can be 
defined as $V_{0}-V$, where $V_{0}$ is the voltage at which $J_{\mathrm{ph}}=0$ and $\mathrm{V}$ is the applied bias. As such, the saturation current $J_{\text {sat }}$ was determined to be 9.26, 9.56, 9.15, 9.88, 9.93 and $9.29 \mathrm{~mA} / \mathrm{cm}^{2}$ for the reference device and the solar cells containing P1, P2, P3, P4 and P6, respectively (Figure 8). From these values, $P$ could be estimated by $J_{\mathrm{ph}} / J_{\text {sat }}$ to be 89.7, 94.4, 98.6, 91.1, 91.7 and $95.5 \%$, respectively, under short-circuit conditions, confirming that the CPE bearing devices show an elevated exciton dissociation rate and an enhanced charge collection efficiency in comparison to the reference device.

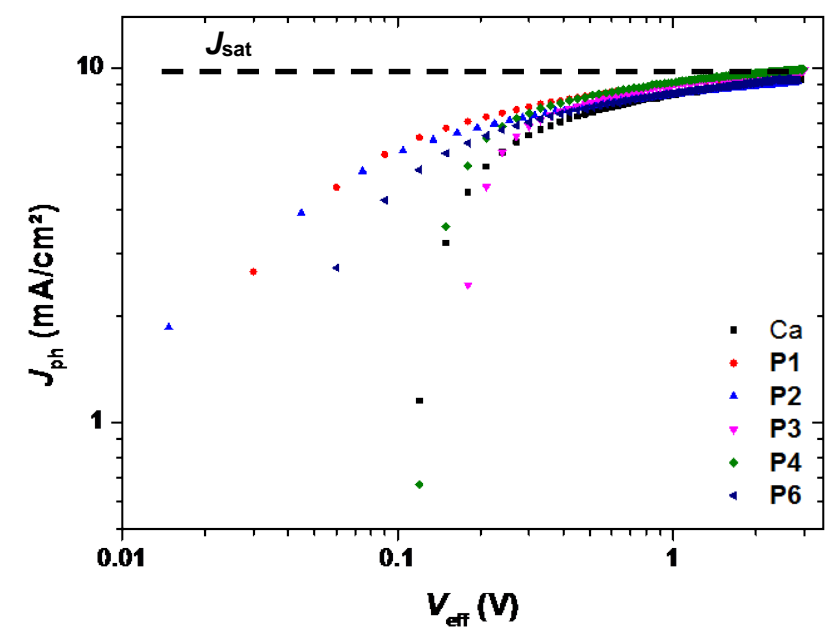

Figure 8. Photocurrent of PCDTBT:PC ${ }_{71} \mathrm{BM}$ photovoltaic devices with and without $\mathrm{CPE}$ cathode interlayers as a function of the effective voltage.

\section{Conclusions}

A series of P3MEEET-co-P3(MIM)HOET-TFSI random copolymers with different ratios of the ionic side chains (30/50/70\%; P2-P4), the homopolymer counterparts P1 and P5, and the 50/50 ionic block copolymer analogue P6 were successfully synthesized via Kumada catalyst-transfer condensation polymerization and subsequent introduction of the ionic moieties and counteranion exchange. Because of the high degree of control that can be achieved in such a polymerization, all copolymers were obtained in the desired monomer ratios and with the targeted molar masses of $\sim 25000 \mathrm{~g} / \mathrm{mol}$. All materials demonstrated 
similar absorption characteristics in solution. On the other hand, the polymer crystallinity clearly increased upon decreasing the amount of ionic groups.

The ionic (co)polythiophenes were then applied as cathode interlayer materials in BHJ photovoltaic devices based on PCDTBT:PC ${ }_{71} \mathrm{BM}$. All photovoltaic parameters ( $\left.V_{\mathrm{OC}}, J_{\mathrm{SC}}, \mathrm{FF}\right)$ improved throughout the complete series in comparison to a reference device employing Ca, pointing to the generality of the efficiency gain (on average $15-17 \%$ ) for this interlayer class, regardless of the structural details. The elevated $J_{\mathrm{SC}}$ was confirmed by determining the charge dissociation probabilities of all devices. These measurements revealed an increase in $P$, and hence the exciton dissociation rate and charge collection efficiency, for all CPE bearing devices. AFM analysis of the final device stacks showed that the polythiophene-based CPE interlayers never afford a completely covered photoactive layer and that the deposition patterns strongly vary with the CPE concentration. The AFM images also illustrated that an improved compatibility for deposition onto the photoactive layer is obtained by increasing the ratio of triethylene glycol-to-ionic side chains. These results confirm our hypothesis that the presence of ionic groups induces the formation of interfacial dipoles, improving the charge collection, while at the same time reducing the affinity of the interlayer material for the photoactive layer, and that the increase in PCE is dependent on the interplay of these two phenomena. ${ }^{23}$ An interesting observation is that the 50/50 block copolymer (slightly) outperforms the 50/50 random copolymer, which previously afforded record efficiencies. ${ }^{23-25}$ Since it is known that control of the morphology is crucial for device performance and that block copolymers can self-assemble into nanostructured morphologies, it seems worthwhile to further explore this route. 


\section{Supporting information}

Instrumentation details, experimental (synthetic) procedures and characterization data as well as ${ }^{1} \mathrm{H}$ NMR, CV, RHC, and TGA figures can be found in the Supporting Information.

\section{Acknowledgments}

This work was supported by the Research Foundation-Flanders (FWO) (project G.0415.14N and postdoctoral fellowship J.K.). S.G. acknowledges Hasselt University for her (BOF) doctoral grant. TA Instruments is acknowledged for the RHC equipment. 


\section{References}

(1) Cao, W.; Xue, J. Recent progress in organic photovoltaics: device architecture and optical design. Energy Environ. Sci. 2014, 7, 2123-2144.

(2) Mazzio, K. A.; Luscombe, C. K. The future of organic photovoltaics. Chem. Soc. Rev. 2015, 44, 78-90.

(3) Wang, K.; Liu, C.; Meng, T.; Yi, C.; Gong, X. Inverted organic photovoltaic cells. Chem. Soc. Rev. 2016, 45, 2937-2975.

(4) Li, S.; Ye, L.; Zhao, W.; Zhang, S.; Mukherjee, S.; Ade, H.; Hou, J. Energy-Level Modulation of Small-Molecule Electron Acceptors to Achieve over 12\% Efficiency in Polymer Solar Cells. Adv. Mater. 2016, 28, 9423-9429.

(5) Yang, Y.; Zhang, Z.-G.; Bin, H.; Chen, S.; Gao, L.; Xue, L.; Yang, C.; Li, Y. SideChain Isomerization on an n-type Organic Semiconductor ITIC Acceptor Makes 11.77\% High Efficiency Polymer Solar Cells. J. Am. Chem. Soc. 2016, 138, 1501115018.

(6) Hu, H.; Jiang, K.; Yang, G.; Liu, J.; Li, Z.; Lin, H.; Liu, Y.; Zhao, J.; Zhang, J.; Huang, F.; Qu, Y.; Ma, W.; Yan, H. Terthiophene-Based D-A Polymer with an Asymmetric Arrangement of Alkyl Chains That Enables Efficient Polymer Solar Cells. J. Am. Chem. Soc. 2015, 137, 14149-14157.

(7) Liu, Y.; Zhao, J.; Li, Z.; Mu, C.; Ma, W.; Hu, H.; Jiang, K.; Lin, H.; Ade, H.; Yan, H. Aggregation and morphology control enables multiple cases of high-efficiency polymer solar cells. Nat. Commun. 2014, 5, 5293.

(8) He, Z.; Zhong, C.; Su, S.; Xu, M.; Wu, H.; Cao, Y. Enhanced power-conversion efficiency in polymer solar cells using an inverted device structure. Nat. Photonics 
2012, 6, 591-595.

(9) Sun, Z.; Xiao, K.; Keum, J. K.; Yu, X.; Hong, K.; Browning, J.; Ivanov, I. N.; Chen, J.; Alonzo, J.; Li, D.; Sumpter, B. G.; Payzant, E. A.; Rouleau, C. M.; Geohegan, D. B. PS- $b$-P3HT Copolymers as P3HT/PCBM Interfacial Compatibilizers for High Efficiency Photovoltaics. Adv. Mater. 2011, 23, 5529-5535.

(10) Seo, J. H.; Gutacker, A.; Sun, Y.; Wu, H.; Huang, F.; Cao, Y.; Scherf, U.; Heeger, A. J.; Bazan, G. C. Improved High-Efficiency Organic Solar Cells via Incorporation of a Conjugated Polyelectrolyte Interlayer. J. Am. Chem. Soc. 2011, 133, 8416-8419.

(11) Kesters, J.; Ghoos, T.; Penxten, H.; Drijkoningen, J.; Vangerven, T.; Lyons, D. M.; Verreet, B.; Aernouts, T.; Lutsen, L.; Vanderzande, D.; Manca, J.; Maes, W. Imidazolium-Substituted Polythiophenes as Efficient Electron Transport Materials Improving Photovoltaic Performance. Adv. Energy Mater. 2013, 3, 1180-1185.

(12) Houston, J. E.; Richeter, S.; Clément, S.; Evans, R. C. Molecular Design of Interfacial Layers based on Conjugated Polythiophenes for Polymer and Hybrid Solar Cells. Polym. Int. 2017, 66, 1333-1348.

(13) Reese, M. O.; White, M. S.; Rumbles, G.; Ginley, D. S.; Shaheen, S. E. Optimal negative electrodes for poly(3-hexylthiophene):[6,6]-phenyl-C61-butyric acid methyl ester bulk heterojunction photovoltaic devices. Appl. Phys. Lett. 2008, 92, 053307.

(14) Ju, H.; Knesting, K. M.; Zhang, W.; Pan, X.; Wang, C.-H.; Yang, Y.-W.; Ginger, D. S.; Zhu, J. Interplay between Interfacial Structures and Device Performance in Organic Solar Cells: A Case Study with the Low Work Function Metal, Calcium. ACS Appl. Mater. Interfaces 2016, 8, 2125-2131.

(15) Gao, M.; Subbiah, J.; Geraghty, P. B.; Chen, M.; Purushothaman, B.; Chen, X.; Qin, 
T.; Vak, D.; Scholes, F. H.; Watkins, S. E.; Skidmore, M.; Wilson, G. J.; Holmes, A.

B.; Jones, D. J.; Wong, W. W. H. Development of a High-Performance

Donor-Acceptor Conjugated Polymer: Synergy in Materials and Device Optimization.

Chem. Mater. 2016, 28, 3481-3487.

(16) Li, G.; Chu, C.-W.; Shrotriya, V.; Huang, J.; Yang, Y. Efficient inverted polymer solar cells. Appl. Phys. Lett. 2006, 88, 253503.

(17) Li, C.-Z.; Chueh, C.-C.; Yip, H.-L.; O’Malley, K. M.; Chen, W.-C.; Jen, A. K.-Y. Effective interfacial layer to enhance efficiency of polymer solar cells via solutionprocessed fullerene-surfactants. J. Mater. Chem. 2012, 22, 8574-8578.

(18) Oh, S.-H.; Na, S.-I.; Jo, J.; Lim, B.; Vak, D.; Kim, D.-Y. Water-Soluble Polyfluorenes as an Interfacial Layer Leading to Cathode-Independent High Performance of Organic Solar Cells. Adv. Funct. Mater. 2010, 20, 1977-1983.

(19) Choi, H.; Park, J. S.; Jeong, E.; Kim, G.-H.; Lee, B. R.; Kim, S. O.; Song, M. H.; Woo, H. Y.; Kim, J. Y. Combination of Titanium Oxide and a Conjugated Polyelectrolyte for High-Performance Inverted-Type Organic Optoelectronic Devices. Adv. Mater. 2011, 23, 2759-2763.

(20) Chang, Y.-M.; Zhu, R.; Richard, E.; Chen, C.-C.; Li, G.; Yang, Y. Electrostatic SelfAssembly Conjugated Polyelectrolyte-Surfactant Complex as an Interlayer for High Performance Polymer Solar Cells. Adv. Funct. Mater. 2012, 22, 3284-3289.

(21) Chevrier, M.; Houston, J. E.; Kesters, J.; Van den Brande, N.; Terry, A. E.; Richeter, S.; Mehdi, A.; Coulembier, O.; Dubois, P.; Lazzaroni, R.; Van Mele, B.; Maes, W.; Evans, R. C.; Clément, S. Self-Assembled Conjugated Polyelectrolyte-Surfactant Complexes as Efficient Cathode Interlayer Materials for Bulk Heterojunction Organic Solar Cells. J. Mater. Chem. A 2015, 3, 23905-23916. 
(22) Liu, H.; Hu, L.; Wu, F.; Chen, L.; Chen, Y. Polyfluorene Electrolytes Interfacial Layer for Efficient Polymer Solar Cells: Controllably Interfacial Dipoles by Regulation of Polar Groups. ACS Appl. Mater. Interfaces 2016, 8, 9821-9828.

(23) Kesters, J.; Govaerts, S.; Pirotte, G.; Drijkoningen, J.; Chevrier, M.; Van den Brande, N.; Liu, X.; Fahlman, M.; Van Mele, B.; Lutsen, L.; Vanderzande, D.; Manca, J.; Clément, S.; Von Hauff, E.; Maes, W. High-Permittivity Conjugated Polyelectrolyte Interlayers for High-Performance Bulk Heterojunction Organic Solar Cells. ACS Appl. Mater. Interfaces 2016, 8, 6309-6314.

(24) Pirotte, G.; Kesters, J.; Verstappen, P.; Govaerts, S.; Manca, J.; Lutsen, L.; Vanderzande, D.; Maes, W. Continuous Flow Polymer Synthesis toward Reproducible Large-Scale Production for Efficient Bulk Heterojunction Organic Solar Cells. ChemSusChem 2015, 8, 3228-3233.

(25) Vanormelingen, W.; Kesters, J.; Verstappen, P.; Drijkoningen, J.; Kudrjasova, J.; Koudjina, S.; Liégeois, V.; Champagne, B.; Manca, J.; Lutsen, L.; Vanderzande, D.; Maes, W. Enhanced open-circuit voltage in polymer solar cells by dithieno[3,2-b:2’,3’d]pyrrole N-acylation. J. Mater. Chem. A 2014, 2, 7535-7545.

(26) Ghoos, T.; Brassinne, J.; Fustin, C.-A.; Gohy, J.-F.; Defour, M.; Van den Brande, N.; Van Mele, B.; Lutsen, L.; Vanderzande, D. J.; Maes, W. Imidazolium-substituted ionic (co)polythiophenes: Compositional influence on solution behavior and thermal properties. Polymer 2013, 54, 6293-6304.

(27) Miyakoshi, R.; Yokoyama, A.; Yokozawa, T. Synthesis of Poly(3-hexylthiophene) with a Narrower Polydispersity. Macromol. Rapid Commun. 2004, 25, 1663-1666.

(28) Thomas, A.; Houston, J. E.; Van den Brande, N.; De Winter, J.; Chevrier, M.; Heenan, R. K.; Terry, A. E.; Richeter, S.; Mehdi, A.; Van Mele, B.; Dubois, P.; Lazzaroni, R.; 
Gerbaux, P.; Evans, R. C.; Clément, S. All-conjugated cationic copolythiophene “rodrod” block copolyelectrolytes: synthesis, optical properties and solvent-dependent assembly. Polym. Chem. 2014, 5, 3352-3362.

(29) Scherf, U.; Gutacker, A.; Koenen, N. All-Conjugated Block Copolymers. Acc. Chem. Res. 2008, 41, 1086-1097.

(30) Ghoos, T.; Van den Brande, N.; Defour, M.; Brassinne, J.; Fustin, C.-A.; Gohy, J.-F.; Hoeppener, S.; Schubert, U. S.; Vanormelingen, W.; Lutsen, L.; Vanderzande, D. J.; Van Mele, B.; Maes, W. Amphiphilic N-methylimidazole-functionalized diblock copolythiophenes. Eur. Polym. J. 2014, 53, 206-214.

(31) Park, S. H.; Roy, A.; Beaupré, S.; Cho, S.; Coates, N.; Moon, J. S.; Moses, D.; Leclerc, M.; Lee, K.; Heeger, A. J. Bulk heterojunction solar cells with internal quantum efficiency approaching 100\%. Nat. Photonics 2009, 3, 297-303.

(32) Zhang, G.; Yang, G.; Yan, H.; Kim, J.-H.; Ade, H.; Wu, W.; Xu, X.; Duan, Y.; Peng, Q. Efficient Nonfullerene Polymer Solar Cells Enabled by a Novel Wide Bandgap Small Molecular Acceptor. Adv. Mater. 2017, 29, 1606054. 